\title{
Posterior reversible encephalopathy syndrome as the initial presentation of acute lymphoblastic leukaemia
}

\author{
Wan Jamaludin W. F. ${ }^{1}$, Mohd Zaki F. ${ }^{2}$, Tumian N.R. ${ }^{3}$, Wong C.L. ${ }^{3}$, Abdul Wahid S.F. ${ }^{3}$ \\ 1. Department of Medicine, University Teknologi MARA (UiTM), Malaysia. 2. Department of Radiology, University \\ Kebangsaan Malaysia Medical Centre (UKMMC), Malaysia. 3. Cell Therapy Centre \& Department of Medicine, University \\ Kebangsaan Malaysia Medical Centre (UKMMC), Malaysia.
}

Correspondnce: Fadilah Abdul Wahid. Address: Cell Therapy Centre \& Department of Medicine, University Kebangsaan Malaysia Medical Centre (UKMMC), Jalan Yaacob Latif, 56000 Kuala Lumpur, Malaysia. Telephone: 60-0391456-392. Fax: 60-039-1456-679. Email: sfadilah@ppukm.ukm.my

Received: June 29, 2011

Accepted: September 9, 2011

Published: December 1, 2011

DOI : $10.5430 /$ jhm.v1n1p49

URL: http://dx.doi.org/10.5430/jhm.v1n1p49

\section{Abstract}

Posterior reversible encephalopathy syndrome (PRES) is a recognized complication of chemotherapy. However, the development of PRES at presentation of acute lymphoblastic leukemia (ALL) is rare. Here we discuss the uncommon presenting feature of PRES in ALL, and its progression with chemotherapy.

A 13-year-old Malay male presented with a one-week history of blurred vision and headache associated with hypertension, hyperkalemia and acute renal failure. CT brain imaging showed symmetrical and bilateral white matter hypodensities at the parietal regions. Brain MRI on T2 and FLAIR images showed patchy areas of high signal intensity at cortical and subcortical region of both parietal and occipital lobes, consistent with PRES. He developed bicytopenia and bone marrow biopsy confirmed pre-B acute lymphoblastic leukemia. He received Phase 1 induction chemotherapy according to the modified UKALL XII protocol but in view of PRES, L-asparaginase and intrathecal methotrexate were delayed until after Day 17 of chemotherapy. No neurological symptoms were observed after chemotherapy. However, during Phase 2 induction chemotherapy with high dose cytarabine and intrathecal methotrexate, he developed accelerated hypertension and status epilepticus requiring mechanical ventilation. Cerebrospinal fluid analysis did not show infection or leukemic infiltration. A repeat brain MRI showed worsening features of the pre-existing PRES, followed by partial resolution on Day 37 of chemotherapy. Subsequent cytotoxic chemotherapies were uneventful except for radiological deterioration of PRES with partial resolution upon hematological recovery.

It is important to consider the diagnosis of PRES in a young patient presenting with acute onset of headache and visual disturbances even before the initiation of cytotoxic chemotherapy. In view of the pre-existing PRES, there are risks of further neurological damage and fatal neurological squeal by introducing chemotherapy to treat the leukemia. Therefore it is vital to recognize and treat PRES promptly as early intervention may prevent or reverse the development of encephalopathy. 


\section{Key words}

Posterior reversible encephalopathy syndrome, Acute lymphoblastic leukemia

\section{I ntroduction}

Posterior reversible encephalopathy syndrome (PRES) is a recognized complication of chemotherapy. However, PRES associated with acute lymphoblastic leukemia (ALL) is rare. A 13-year-old Malay male presented with headache, vomiting, reduced vision, papilledema and accelerated hypertension. MRI brain on T2 and fluid attenuated inversion recovery (FLAIR) images showed hyperintensity signal at parieto-occipital lobes consistent with PRES. He developed bicytopenia and bone marrow biopsy confirmed pre-B acute lymphoblastic leukemia. He developed status epilepticus and worsening brain MRI features requiring mechanical ventilation following cytotoxic chemotherapy with high dose cytarabine and intrathecal methotrexate. Subsequent chemotherapies were uneventful except for radiological deterioration of PRES with partial resolution upon hematological recovery. In agreement with other reports [1], our case illustrates that the term posterior reversible encephalopathy syndrome is a misnomer as it is not always reversible.

\section{Case presentation}

A previously healthy 13-year-old Malay male presented with one week history of blurred vision, headache, vomiting and upper limb and lower limb weakness with power reduction of 4 out of 5. Cranial nerves were intact. Renal profiles were impaired with urea $38.6 \mathrm{mmol} / \mathrm{L}$, creatinine $374 \mu \mathrm{mol} / \mathrm{L}$, and hypercalcemia $4.12 \mathrm{mmol} / \mathrm{L}$ with hyperphosphatemia 2.29 $\mathrm{mmol} / \mathrm{L}$. Parathyroid hormone was suppressed. Magnesium level and full blood count on admission was normal. Autoimmune screen and tumor markers were negative. Blood pressure was elevated to $165 / 104 \mathrm{mmHg}$ despite no history of previous hypertension. Fundoscopy showed papilledema but no associated silver wiring or retinal hemorrhages. The hypertension was controlled with calcium antagonists and alpha-blockers initially whilst investigating for secondary hypertension. Urinary catecholamine, plasma renin and aldosterone were normal with no radiological evidence of paraganglioma, adrenal tumors or renal artery stenosis. Total body CT scan revealed bilateral pleural effusion, ascites, hepatomegaly and multiple lytic bony lesions. CT brain showed bilateral symmetrical white matter hypodensities in the parietal regions. MRI brain showed high signal intensity on T2 and FLAIR images at cortical and subcortical region of parieto-occipital lobes bilaterally (Figure 1). These findings supported the diagnosis of PRES. He developed bicytopenia (platelets $56 \times 10^{9} / \mathrm{L}$, hemoglobin $10.1 \mathrm{~g} / \mathrm{dL}$, white cells $6.6 \times 10^{9} / \mathrm{L}$ ). Bone marrow biopsy showed pre-B acute lymphoblastic leukemia (ALL) with 28\% blasts expressing CD34, HLA-DR, CD19, CD10, CD22 (dim) with aberrant CD33 (dim) and CD13. The blasts were negative for CD79a, CD20 and other myeloid and T lymphoid markers. He received modified Phase 1 MRC UKALL XII standard risk chemotherapy protocol with prednisolone $60 \mathrm{mg} / \mathrm{m}^{2}$, vincristine $1.4 \mathrm{mg} / \mathrm{m}^{2}$, daunorubicin $30 \mathrm{mg} / \mathrm{m}^{2}$, L-asparaginase $10,000 \mathrm{units} / \mathrm{m}^{2}$ and intrathecal methotrexate $12.5 \mathrm{mg}$. CSF examination was unremarkable. A second brain MRI 20 days after Phase 1 induction demonstrated resolution of PRES.

He received Phase 2 induction high risk chemotherapy protocol with high dose cytarabine $1 \mathrm{~g} / \mathrm{m}^{2}$ and intrathecal methotrexate $12.5 \mathrm{mg}$. The adjustment to the chemotherapy regimen was made after taking the atypical clinical presentation into consideration. CSF examinations were unremarkable. He developed febrile neutropenia and concurrently the previously controlled hypertension rapidly elevated to $158 / 114 \mathrm{mmHg}$ followed by a generalized seizure. Within the next three hours he developed two further seizures requiring mechanical ventilation. MRI brain 
showed worsening of PRES in the parieto-occipital lobes (Figure 2). No evidence of meningitis or cerebral abscess was observed. CSF examination was normal. GCS fluctuated between 8 and 12 associated with episodes of emotional lability and inability to focus. Despite reactive pupils he had no blinking response to movements or light stimuli, raising the possibility of cortical blindness. Formal eye assessment was uninformative due to the lack of verbal response. EEG demonstrated severe cerebral disturbance with epileptic discharges. Over the next few days he sustained further multiple seizures with systolic pressure up to $200 \mathrm{mmHg}$. Clinical improvement was observed following optimum control of blood pressure with calcium antagonists and addition of beta-blockers in place of alpha-blockers. He regained full consciousness and normalization of visual function.

He was maintained on phenytoin and sodium valproate. Brain MRI five weeks after Phase 2 induction showed reduction of the parieto-occipital hyperintensity signals with new evidence of focal infarct with hemorrhage in the right occipital region. He continued Phase 3 induction with high dose methotrexate $2.5 \mathrm{gram} / \mathrm{m}^{2}$ and L-asparaginase 10,000 units $/ \mathrm{m}^{2}$ without intrathecal methotrexate. Due to refractory disease, he received FLAG chemotherapy (fludarabine $25 \mathrm{mg} / \mathrm{m}^{2}$, cytarabine $2 \mathrm{~g} / \mathrm{m}^{2}$, GCSF $5 \mu \mathrm{g} / \mathrm{kg}$ ) with no neurological symptoms. However brain MRI eleven days after FLAG chemotherapy showed extensive parieto-occipital white matter edema. CSF examination excluded leukemic infiltration.

A follow-up brain MRI three weeks later showed reduction of the parieto-occipital white matter hyperintensities although there were residual patchy hyperintensities in the subcortical white matter. Nine months after the initial presentation, brain MRI showed complete remission of the high signal intensity in both occipital lobes, in keeping with complete resolution of PRES. However, the lesions in both parietal regions have become more prominent (Figure 3).

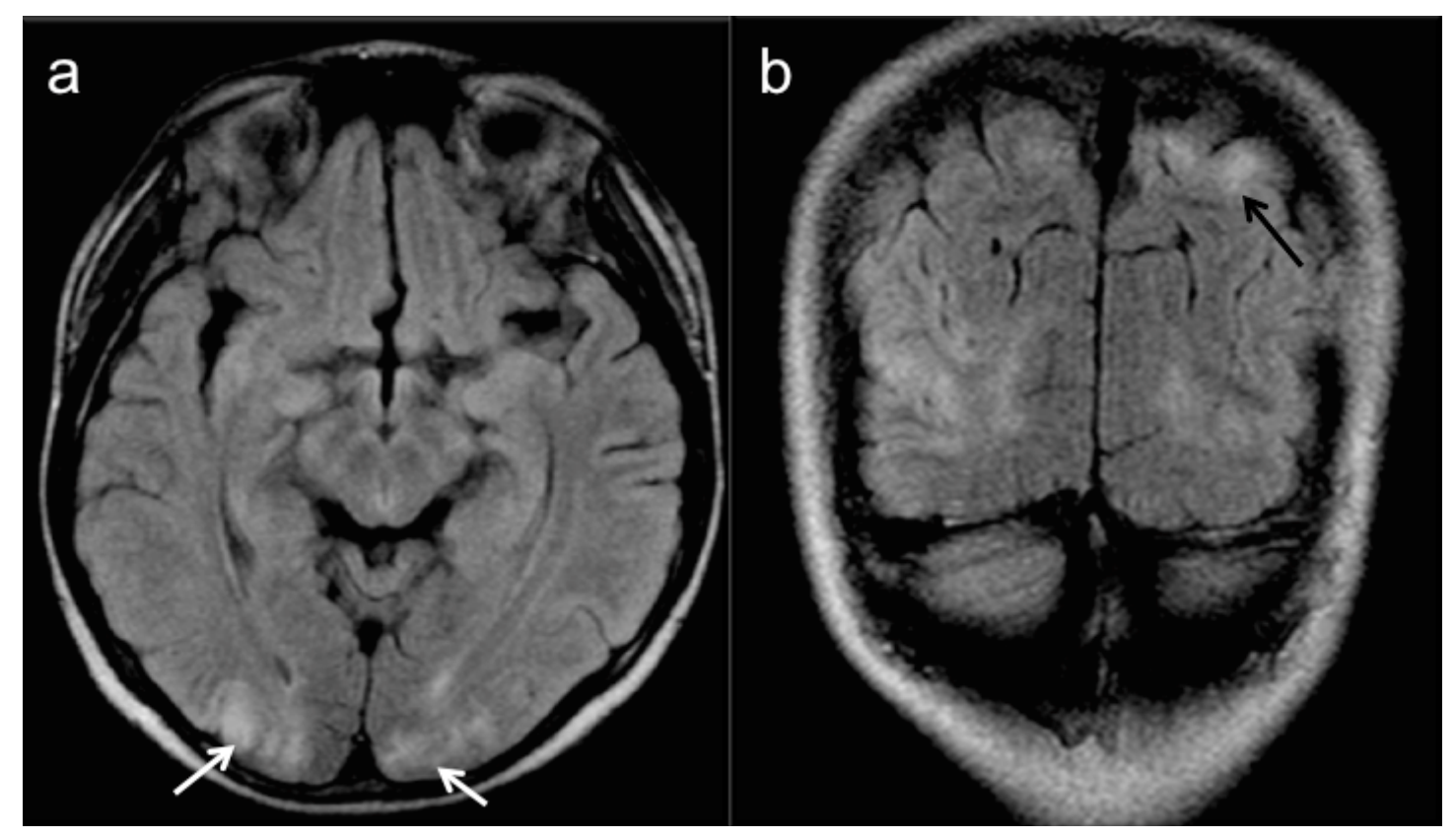

Figure 1. Brain MRI T2-FLAIR images at first presentation of ALL showing high intensity signals in the occipital lobes (white arrows) and in the cortex of left parietal lobe (black arrow) which support the diagnosis of PRES. (a) Axial view (b) Coronal view 


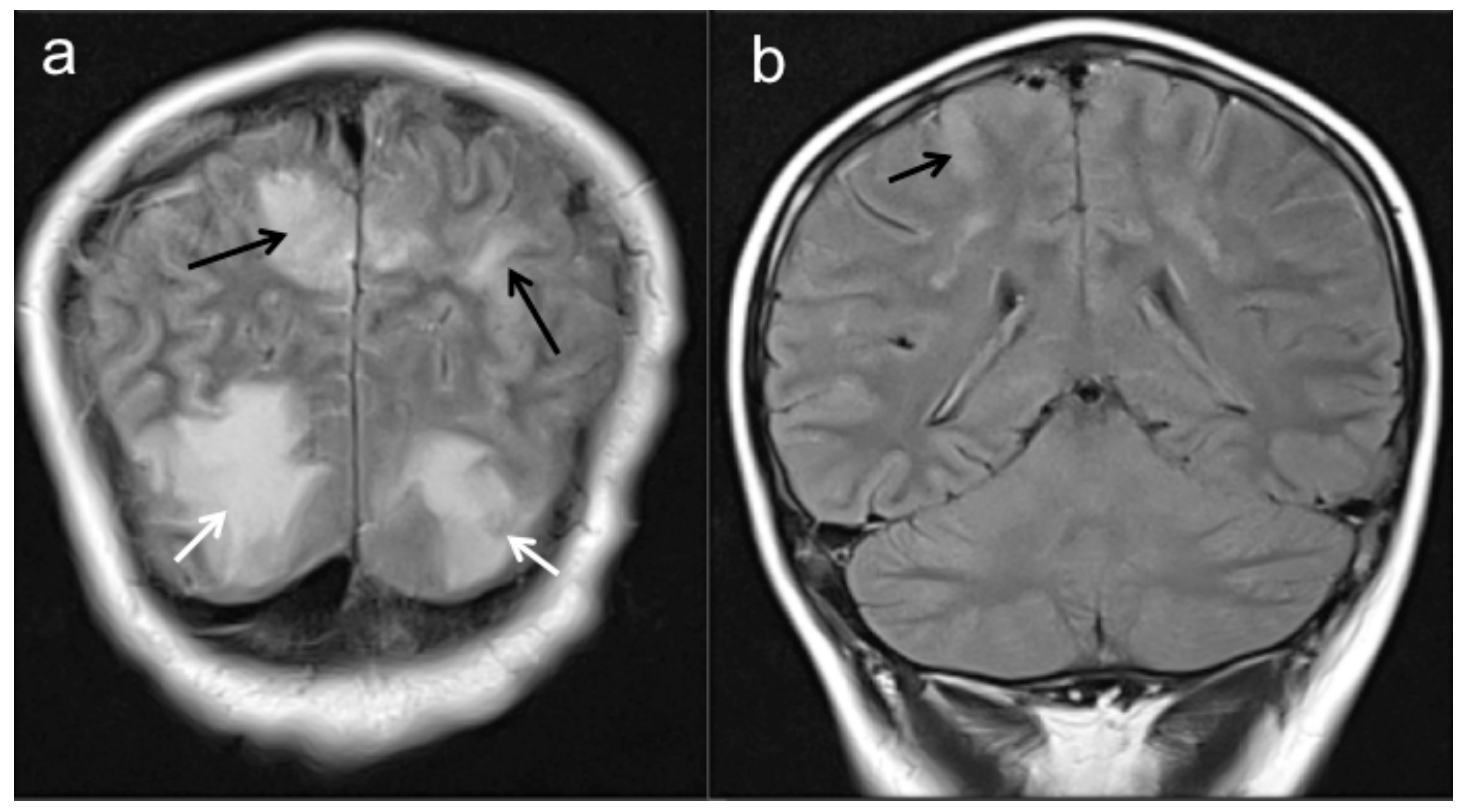

Figure 2. Brain MRI images after high dose cytarabine and intrathecal methotrexate showing new hyperintense lesions in both parietal (black arrows) and occipital (white arrows) lobes, which are worse than the first MRI (Figure 1). (a) T2WI. (b) T2-FLAIR

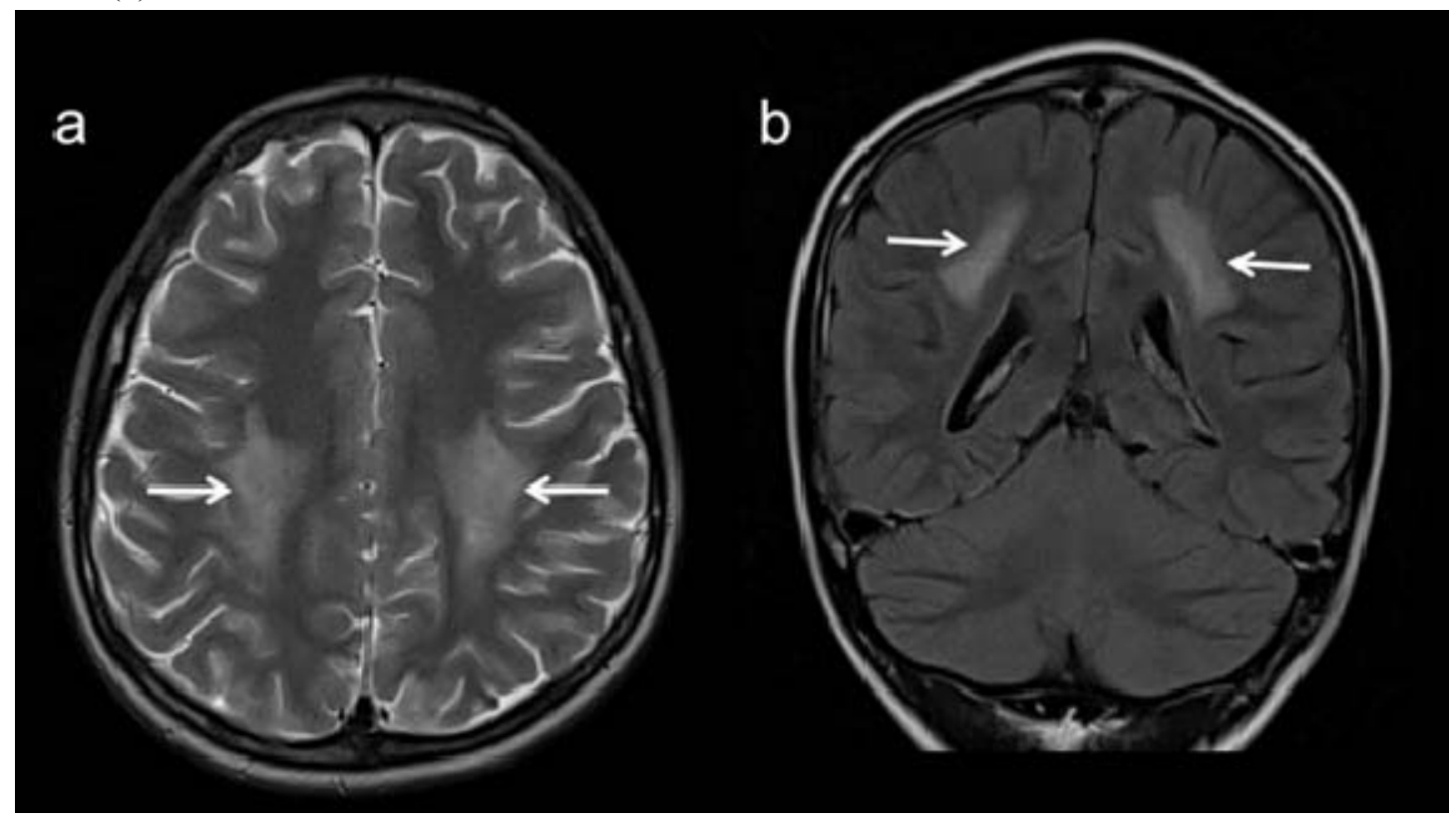

Figure 3. Post therapy brain MRI showing more prominent lesions in both parietal lobes as compared to Figure 2 (white arrows). The previously seen lesions in occipital lobes (Figure 2) have resolved. (a) Axial T2WI at level of corona radiata. (b) Coronal T2 FLAIR at level of trigone of lateral ventricle

\section{Discussion}

We highlight the occurrence of PRES as the initial presentation of ALL in a young patient before initiation of cytotoxic chemotherapy and the neurological sequelae that developed after commencement of chemotherapy. 
PRES is a clinical-radiological entity with constellation of headache, vomiting, altered mental function, seizures and visual abnormalities [2]. The onset is usually subacute but may be heralded by a seizure. Hypertension is commonly present. PRES is associated with hypertensive encephalopathy, uremic encephalopathy, systemic lupus erythematosus, eclampsia, immunosuppressive therapy and tumor lysis syndrome $[2,3]$. The exact mechanism is uncertain, but the development of cerebral edema in the pathophysiology may be explained by the vasogenic and cytotoxic hypothesis. Sudden elevations in systemic blood pressure may exceed cerebral vascular autoregulation, causing vasodilatation, capillary leakage, acute disruption of the blood brain barrier and vasogenic edema [2, 4]. Another possibility is sudden and severe increase in blood pressure may cause cerebral vasoconstriction, causing ischemia and cytotoxic edema. Endothelium damage caused by direct drug toxicity may also lead to endothelium dysfunction, reduced tissue perfusion, activation of the coagulation cascade and fluid extravasation [4]. Classically PRES is observed in the posterior cerebral circulation. The reason for this is poorly understood. It is possible that cerebral sympathetic innervation is weaker posteriorly $[2,5]$, where it is more susceptible to the development of vasogenic edema as autoregulation fails. Less commonly, frontal and temporal lobes, the brainstem, basal ganglia, and cerebellum may also be involved [6].

MRI is the preferred modality of imaging as it is more sensitive than CT. The involvement of PRES is usually bilateral and symmetrical and is seen as an increased signal on MRI T2 and FLAIR images [7] and typically involves the occipital lobes. These abnormalities are substantially reversible with prompt and appropriate therapy consistent with the proposal that PRES is a result of transient vasogenic edema rather than infarction [2, 7]. However, the term reversible posterior encephalopathy can be a misnomer as the condition is not always reversible [1], and is not necessarily confined to the posterior regions of the brain and can affect both white and grey matter even with adequate therapy [4]. Atypical radiological findings such as hemorrhage, infarction and extensive edema may predict non-reversibility and a poorer outcome. Additionally, prolonged seizures or hypertension may result in permanent neurological damage and cerebral infarction [7, 8]. A focal right external capsule lesion representing an infarct was present during the initial brain MRI upon presentation in our patient. Subsequently, new acute infarcts in both frontal lobes and lacunar infarcts in the pons were identified on MRI scan after he received high dose cytarabine and intrathecal methotrexate. Micro bleeding in the parietal lobes was also reported on MRI scan eleven days after FLAG chemotherapy.

PRES as an initial presenting feature of hematological malignancies is rare in comparison to its occurrence after initiation of cytotoxic therapy. The uncommon presenting feature of PRES was reported in a 50-year-old female with Burkitt lymphoma who developed hypercalcemia of $3.91 \mathrm{mmol} / \mathrm{L}$ without hypertension [9]. CSF examination excluded lymphoma infiltration and brain MRI was consistent with typical findings of symmetrical posterior cortical and subcortical lesions which resolved completely after treatment. In ALL, the incidence of PRES is more commonly observed after induction chemotherapy, possibly due to synergistic neurotoxicity of multiple chemotherapeutic agents. Hypertension and cytotoxic chemotherapy were identified as the predisposing factors for PRES occurring after induction chemotherapy in eleven children between 5 to 16 years with acute myeloid leukemia, ALL, Non-Hodgkin lymphoma and Ewing sarcoma [1]. Long term sequelae occurred with irreversible MRI abnormalities in four children, epilepsy in three, and continued abnormal EEG without further seizures in one. Intensive chemotherapy was also identified as a risk factor in two young patients with ALL who developed PRES following acute renal failure, hypertension and seizures during GRALL-2005 induction chemotherapy [10]. A 9-year retrospective analysis of central nervous system (CNS) complications in patients with ALL in a single Italian pediatric institution found PRES being the most frequent neurological complication following treatment with the AIEOP-BFM-ALL-2000 protocol [11]. PRES also occurred in two patients with ALL following multidrug induction chemotherapy with vincristine, daunomycin, prednisone, Lasparaginase and intrathecal methotrexate [12], where the second patient also developed tumor lysis syndrome immediately after chemotherapy. Tumor lysis syndrome is implicated in PRES, most likely due to the release of intracellular components leading to acute renal failure and systemic hypertension $[3,13]$.

With regards to chemotherapy agents, cytarabine and methotrexate given orally, parenterally or intrathecally are implicated with PRES [14]. Cytarabine administered intrathecally is generally reported with PRES rather than when 
administered by the intravenous route, although cytarabine rapidly distributes into the CSF and when given intravenously in high doses, it could potentially causes neurotoxicity [15]. Methotrexate is capable of crossing the blood brain barrier and may have caused neurotoxicity via biochemical and metabolic alterations [1]. PRES was reported in a 15-year-old girl with ALL who developed status epilepticus without hypertension, 10 days after her fourth intrathecal methotrexate [16]. However this patient had also received multidrug induction chemotherapy with dexamethasone, vincristine, daunoblastine, L-asparaginase, and cytarabine. Although PRES is an uncommon cause of methotrexate related neurorotoxicity, the authors concluded that the development of brain vasogenic edema in this patient was likely a final event of a direct toxic derangement resulting from microangiopathic damage to the brain vascular endothelium, leading to focal blood brain barrier impairment. CNS complications secondary to L- asparaginase such as hemorrhage, cerebral venous thrombosis and thromboemboli are common but L-asparaginase induced cerebral or cerebellar lesions are rare [17]. PRES was reported in 3 children with ALL who developed seizures and altered sensorium after Lasparaginase therapy [18]. The first patient developed seizures during the first week maintenance with dexamethasone $1 \mathrm{mg} / \mathrm{m} 2$ and L-asparaginase 25000 units $/ \mathrm{m}^{2}$, the second patient had seizures after 2 hours of receiving third dose of Lasparaginase for re-induction for relapsed ALL while the third developed paraesthesia and myoclonic movements after one week from the last dose of L-asparaginase. All three cases showed increased T2 and FLAIR signal intensity in the posterior regions of the brain whilst brain CT and brain MR venography excluded hemorrhage and venous sinus thrombosis. The authors concluded the occurrence of brain lesions and acute clinical deterioration directly after Lasparaginase administration suggested that the brain changes are secondary to L-asparaginase toxicity. Brain MRI performed one month later on the first patient showed complete resolution of the PRES changes after discontinuation of L-asparaginase but no follow up MRI were performed on the other two patients as they deteriorated from septic shock.

Our patient developed accelerated hypertension, multiple seizures and radiological deterioration of PRES after receiving high dose cytarabine and intrathecal methotrexate. These drugs may have caused neurotoxicity by damaging the blood brain barrier or via direct toxicity on the vascular endothelium. Subsequent chemotherapies were uneventful except for radiological deterioration of PRES followed by its partial resolution, as evidenced by serial MRI scans. Lumbar punctures excluded leukemia infiltration as a cause for the deterioration. This indicated that the pre-existing PRES was made worse by administration of combination chemotherapy and resolved as the patient recovered from chemotherapy.

The mechanism of PRES at presentation of ALL before the initiation of cytotoxic chemotherapy in our patient was likely to be multifactorial. Our patient presented with severe hypercalcemia, acute renal failure and hypertension. It may begin with hypercalcemia due to osteolytic lesions which is uncommon in ALL, secondary to secretion of parathyroid hormone, or parathyroid hormone related peptide from the lymphoblasts [19], or osteoclastic bone resorption driven by proinflammatory cytokines [20]. PRES associated with hypercalcemia was also observed in patients with calcium overdose and plasmacytoma [21]. Hypercalcemia can cause smooth muscle dysfunction with arterial media vasoreactivity and impaired endothelium-independent vasodilation [22]. Therefore, ensuing vasoconstriction in the renal and cerebral circulations may lead to renal insufficiency and PRES, respectively. Subsequent renal impairment may have caused worsening of the underlying hypertension.

PRES must be suspected in a context of neurological deterioration with progressive hypercalcemia and hypertension. Delaying or moderating chemotherapy are not favorable measures in newly diagnosed ALL, however the potential significant neurotoxicity caused by chemotherapy on top of the background of pre-existing PRES can be associated with significant morbidity. Patients with PRES should undergo sequential MRI to detect radiological progression during the course of treatment. If these patients are in clinical remission, the use of less intensive chemotherapy regimen may be favorable. PRES is not always fully reversible and its location is not necessarily confined to the posterior circulation. Nonetheless, early recognition of PRES, prompt treatment of hypertension, use of anticonvulsants and avoiding of the offending drugs may improve outcome and prevent irreversible cerebral damage. Our patient reported no further seizures and is currently on antiepileptic medications for a total of six months. 


\section{Acknowledgements}

Manuscript writing: Dr.Wan Fariza Wan Jamaludin, Dr. Nur Rafeah Tumian, Professor Dr. Fadilah S. Abdul Wahid Interpretation and Provision of MRI images: Dr. Faizah Mohd Zaki

Review of manuscript: Associate Professor Dr. Wong Chieh Lee

Final approval of manuscript: Dr. Wan Fariza Wan Jamaludin, Dr. Faizah Mohd Zaki, Dr. Nur Rafeah Tumian, Associate Professor Dr. Wong Chieh Lee, Professor Dr. Fadilah S. Abdul Wahid

\section{Conflict of interest}

We declare that we have no conflict of interest.

\section{References}

[1] Laningham F.H., Kun L.E., Reddick W.E., Ogg R.J., Morris E.B., Pui C.H. Childhood central nervous system leukemia: historical perspectives, current therapy, and acute neurological sequelae. Neuroradiology. 2007;49:873-88. PMid: 17924103. http://dx.doi.org/10.1007/s00234-007-0300-7

[2] Hinchey J., Chaves C., Appignani B., Breen J., Pao L., Wang A., Pessin M.S., Lamy C., Mas J.L., Caplan L.R. A reversible posterior leukoencephalopathy syndrome. New England Journal of Medicine. 1996;334:494-500. PMid: 8559202. http://dx.doi.org/10.1056/NEJM199602223340803

[3] Ozkan A., Hakyemez B., Ozkalemkas F., Ali R., Ozkocaman V., Ozcelik T., Taskapilioglu O., Altundal Y., Tunali A. Tumour lysis syndrome as a contributory factor to the development of reversible posterior leukoencephalopathy. Neuroradiology. 2006;48:887-92. PMid: 16983525. http://dx.doi.org/10.1007/s00234-006-0142-8

[4] Stott V.L., Hurrell M.A., Anderson T.J. Reversible posterior leukoencephalopathy syndrome: a misnomer reviewed. Internal Medicine Journal. 2005;35:83-90. PMid: 15705136. http://dx.doi.org/10.1111/j.1445-5994.2004.00750.x

[5] Ay H., Buonanno F.S., Schaefer P.W., Le D.A., Wang B., Gonzalez R.G., Koroshetz W.J. Posterior leukoencephalopathy without severe hypertension: utility of diffuse-weighted MRI. Neurology. 1998;51:1369-76.

[6] Ugurel M.S., Hayakawa M. Implications of post-gadolinium MRI results in 13 cases with posterior reversible encephalopathy syndrome. European Journal of Radiology. 2005;53:441-49. PMid: 15741018 http://dx.doi.org/10.1016/j.ejrad.2004.05.015

[7] Gumus H., Per H., Kumandas S., Yikilmaz A. Reversible posterior leukoencephalopathy syndrome in childhood: report of nine cases and review of the literature. Neurological Sciences. 2010;31:125-31. PMid: 19809787. http://dx.doi.org/10.1007/s10072-009-0158-z

[8] Prasad N., Gulati S., Gupta R.K., Kumar R., Sharma K., Sharma R.K. Is reversible posterior leukoencephalopathy with severe hypertension completely reversible in all patients? Pediatric Nephrology. 2003;18:1161-66. PMid: 14505162. http://dx.doi.org/10.1007/s00467-003-1243-9

[9] Ma E.S.K, Chiu E.K.W., Fong G.C.Y., Li F.K, Wong C.L.P. Burkitt lymphoma presenting as posterior reversible encephalopathy syndrome secondary to hypercalcaemia. British Journal of Haematology. 2009;146:584. PMid: 19344407. http://dx.doi.org/10.1111/j.1365-2141.2009.07629.x

[10] Reutenauer S., Albucher J.F., Pariente J., Dumas H., Milioto O., Attal M., Recher C., Huguet F. Posterior reversible encephalopathy syndrome: Two cases in young adults with acute lymphoblastic leukaemia. Leukaemia Research. 2009;33:e1-e3. PMid: 18922579. http://dx.doi.org/10.1016/j.leukres.2008.09.003

[11] Parasole R., Petruzziello F., Menna G., Mangione A., Cianciulli E., Buffardi S., Marchese L., Nastro A., Misuraca A., Poggi V. Central nervous system complications during treatment of acute lymphoblastic leukaemia in a single pediatric institution. Leukaemia \& Lymphoma. 2010;51(6):1063-71. http://dx.doi.org/10.3109/10428191003754608

[12] Gupta A., Swaroop C., Rastogi R., Garg R., Bakhshi S. Simultaneous occurrence of posterior reversible leukoencephalopathy syndrome in two cases of childhood acute lymphoblastic leukaemia induction chemotherapy. Pediatric Hematology and Oncology. 2008;25:351-38. PMid: 18484481. http://dx.doi.org/10.1080/08880010802016052

[13] Kaito E., Terae S., Kobayashi R., Kudo K.,Tha K.K., Miyasaka K. The role of tumour lysis in reversible posterior leukoencephalopathy syndrome. Pediatric Radiology. 2005;35:722-27. PMid: 15756541. http://dx.doi.org/10.1007/s00247-005-1434-6

[14] Shah-Khan F.M., Pinedo D., Shah P. Reversible posterior leukoencephalopathy syndrome and anti-neoplastic agents: a review. Oncology Reviews. 2007;1:152-61. http://dx.doi.org/10.1007/s12156-007-0018-0 
[15] Henderson R.D., Rajah T., Nicol A.J., Read S.J. Posterior leukoencephalopathy following intrathecal chemotherapy with MRAdocumented vasospasm. Neurology. 2003;60:326-28. PMid: 12552054.

[16] Dicuonzo F., Salvati A., Palma M., Lefons V., Lasalandra G., De Leonardis F., Santoro N. Posterior reversible encephalopathy syndrome associated with methotrexate neurotoxicity: conventional magnetic resonance and diffusion-weighted imaging findings. Journal of Child Neurology. 2009;24:1013-18. PMid: 19307676. http://dx.doi.org/10.1177/0883073809332705

[17] Kieslich M., Porto L., Lanfermann H., Jacobi G., Schwabe D., Bohles H. Cerebrovascular complications of L-asparaginase in the therapy of acute lymphoblastic leukaemia. Journal of Pediatric Hematology / Oncology. 2003;25:484-87. PMid: 12794528. http://dx.doi.org/10.1097/00043426-200306000-00011

[18] Hourani R., Abboud M., Hourani M., Khalifeh H., Muwakkit S. L-asparaginase induced posterior reversible encephalopathy syndrome during acute lymphoblastic leukaemia treatment in children. Neuropediatrics. 2008;39:46-50. PMid: 18504683. http://dx.doi.org/10.1055/s-2008-1076740

[19] Ramsay N.K.S., Brown D.M., Nesbit M.E., Coccia P.F., Krivit, W., Krutzik S. Autonomous production of parathyroid hormone by lymphoblastic leukaemia cells in culture. J Paediatr. 1979,94:623-25. http://dx.doi.org/10.1016/S0022-3476 (79)80034-7

[20] Desmond R.,McDerra J., Kelly K., Smith O. Multiple vertebral collapse as a presentation of childhood acute lymphoblastic leukaemia. British Journal of Haematology. 2009;144:627. PMid: 19006561 http://dx.doi.org/10.1111/j.1365-2141.2008.07396.x

[21] Kastrup O., Maschke M., Wanke I., Diener H.C. Posterior reversible encephalopathy syndrome due to severe hypercalcaemia. Journal of Neurology. 2002;249:1563-1566. PMid: 12420098. http://dx.doi.org/10.1007/s00415-002-0895-x

[22] Neunteufl T., Katzenschlager R., Abela C., Kostner K., Niederle B., Weidinger F., Stefenell T. Impairment of endotheliumindependent vasodilation in patients with hypercalcaemia. Cardiovascular Research. 1998;40:396-401. PMid: 9893734. 